QUARTERLY OF APPLIED MATHEMATICS

VOLUME LXIX, NUMBER 1

MARCH 2011, PAGES 39-55

S 0033-569X(2010)01190-1

Article electronically published on December 30, 2010

\title{
GLOBAL EXISTENCE IN NONLINEAR HYPERBOLIC THERMOELASTICITY WITH RADIAL SYMMETRY
}

\author{
BY
}

\author{
TILMAN IRMSCHER
}

Department of Mathematics and Statistics, University of Konstanz, Germany

\begin{abstract}
In this paper we consider a nonlinear system of hyperbolic thermoelasticity in two or three dimensions with DIRICHLET boundary conditions in the case of radial symmetry. We prove the global existence of small, smooth solutions and the exponential stability.
\end{abstract}

1. Introduction. The equations of thermoelasticity are used to model the behaviour of elastic and heat conductive media. Let $u=u(t, x), \vartheta=\vartheta(t, x)$, and $q=q(t, x)(t \geq 0$, $x \in \Omega, \Omega \subset \mathbb{R}^{n}$ bounded) be the displacement vector, the temperature difference to a fixed reference temperature, and the heat flux, respectively; then the linear differential equations for $(u, \vartheta, q)$ are first

$$
\begin{aligned}
u_{t t}-\alpha \Delta u+\beta \nabla \vartheta=0 & \text { in }[0, \infty) \times(0, L), \\
\zeta \vartheta_{t}+\gamma \operatorname{div} q+\beta \operatorname{div} u_{t}=0 & \text { in }[0, \infty) \times(0, L),
\end{aligned}
$$

where (1.1a) is an equation of motion and (1.1b) describes the conservation of energy. The positive coefficients $\alpha, \beta, \zeta, \gamma$ depend on the material. For a physical derivation of (1.1) we refer to 22.

These two equations have to be completed by a heat equation. We use CATTANEO's law of heat propagation

$$
\tau q_{t}+q+\kappa \nabla \vartheta=0 \quad \text { in }[0, \infty) \times(0, L)
$$

with positive constants $\kappa, \tau$. The system (1.1)-(1.2) is purely hyperbolic, but slightly damped, and it models thermal disturbances as wave-like pulses propagating with finite speed, the so-called second sound. For a review of recent literature to the system of hyperbolic thermoelasticity we refer to 4 .

If we use FOURIER's law

$$
q+\kappa \nabla \vartheta=0 \quad \text { in }[0, \infty) \times(0, L),
$$

Received April 8, 2009.

2000 Mathematics Subject Classification. Primary 74F05, 74H40.

E-mail address: tilman.irmscher@web.de

(C)2010 Brown University

Reverts to public domain 28 years from publication 
instead of (1.2) we get the (hyperbolic-)parabolic system of classical thermoelasticity including the paradox of infinite propagation speed of heat pulses.

The system of nonlinear parabolic thermoelasticity with DIRICHLET boundary conditions in two or three space dimensions has been investigated in [9] in view of global existence of small, smooth solutions and exponential decay. Therein, particularly radial symmetry has been studied. As proved in [13, these results can be carried over to some other boundary conditions.

Tarabek 14 as well as Racke 11 then used CATtaneO's law of heat conduction instead of the classical (i.e. FouRIER's) law and discussed the now purely hyperbolic system in the one-dimensional, nonlinear case. It is also well known that under certain conditions the linear hyperbolic system in two or three - actually in all-space dimensions is exponentially stable; cf. [11, 12]. For the multidimensional nonlinear hyperbolic system there are no comparable results on the global existence or exponential stability. This work shall close this gap for space dimensions $n=2,3$ in the radially symmetric case.

We do not want to give a derivation of the nonlinear equations. We rather refer to the mentioned papers and the cited literature therein. Then we want to consider the following nonlinear differential equations for $(u, \vartheta, q)$ :

$$
\begin{aligned}
& u_{i|t| t}-A_{i j}(\nabla u, \vartheta, q) u_{j|k| k}+B_{i j}(\nabla u, \vartheta, q) \vartheta_{\mid j}=0 \quad \text { in } \quad[0, \infty) \times \Omega, \\
& c(\nabla u, \vartheta, q) \vartheta_{\mid t}+g(\nabla u, \vartheta, q) q_{i \mid i}+B_{i j}(\nabla u, \vartheta, q) u_{i|j| t}=0 \quad \text { in } \quad[0, \infty) \times \Omega \text {, } \\
& T_{i j}(\nabla u, \vartheta) q_{j \mid t}+q_{i}+K_{i j}(\nabla u, \vartheta) \vartheta_{\mid j}=0 \quad \text { in } \quad[0, \infty) \times \Omega,
\end{aligned}
$$

with the initial data

$$
u(0)=u^{0}, \quad u_{t}(0)=u^{1}, \quad \vartheta(0)=\vartheta^{0}, \quad q(0)=q^{0},
$$

and the Dirichlet boundary conditions

$$
\left.u\right|_{\partial \Omega}=\left.\vartheta\right|_{\partial \Omega}=0 .
$$

It is self-evident that (1.4a) and (1.4c) hold for all $i=1, \ldots, n$. Also note that we use the EINSTEIN summation convention, i.e. repeated indices are implicitly summed over. This shortens for example the product of matrices $A, B$ to $(A B)_{i j}=A_{i k} B_{k j}$. Finally, we denote the partial derivative $\partial_{i}(\ldots)$, and $\partial_{t}(\ldots)=(\ldots)_{t}$ with $(\ldots)_{\mid i}$, and $(\ldots)_{\mid t}$, respectively.

REMARK 1.1. For more generality, one would use $C_{i j k l}(\nabla u, \vartheta, q) u_{j|k| l}$ in (1.4a) instead of the LAPLACIAN $A_{i j}(\nabla u, \vartheta, q) u_{j|k| k}$. However, this restriction has turned out to be technically very helpful in 9 as well as in this paper.

The appearing coefficients are subject to the following conditions:

Assumption 1.2. Let $A, B, c, g, T$, and $K$ be smooth functions. Assume $A, T$, and $K$ to be symmetrical matrices and that there are positive constants $A_{0}, c_{0}, g_{0}, T_{0}, K_{0}$, and $\varrho$ such that

$$
\begin{array}{lll}
A(\zeta, \eta, \chi) \xi \cdot \xi \geq A_{0}|\xi|^{2}, & c(\zeta, \eta, \chi) \geq c_{0}, & T(\zeta, \eta) \xi \cdot \xi \geq T_{0}|\xi|^{2}, \\
B(\zeta, \eta, \chi) \xi \cdot \xi \neq 0, & g(\zeta, \eta, \chi) \geq g_{0}, & K(\zeta, \eta) \xi \cdot \xi \geq K_{0}|\xi|^{2}
\end{array}
$$

for all $\zeta \in \mathbb{R}^{n \times n}, \eta \in \mathbb{R}, \chi \in \mathbb{R}^{n}$ with $|\zeta|,|\eta|,|\chi|<\varrho$, and $\xi \in \mathbb{R}^{n} \backslash\{0\}$.

Furthermore, we want to regard the nonlinear system as a perturbation of the isotropic 
linear one; i.e. some constants $\alpha, \beta, \zeta, \gamma, \tau$, and $\kappa$ exist with

$$
\begin{array}{lll}
A(0,0,0)=\alpha E_{n}, & c(0,0,0)=\zeta, & T(0,0)=\tau E_{n}, \\
B(0,0,0)=\beta E_{n}, & g(0,0,0)=\gamma, & K(0,0)=\kappa E_{n} .
\end{array}
$$

Hence, we can rewrite (1.4) to

$$
\begin{aligned}
u_{t t}-\alpha \Delta u+\beta \nabla \vartheta & =F, \\
\zeta \vartheta_{t}+\gamma \operatorname{div} q+\beta \operatorname{div} u_{t} & =G, \\
\tau q_{t}+q+\kappa \nabla \vartheta & =H,
\end{aligned}
$$

with

$$
\begin{aligned}
F:= & (A(\nabla u, \vartheta, q)-A(0,0,0)) \Delta u-(B(\nabla u, \vartheta, q)-B(0,0,0)) \nabla \vartheta, \\
G:= & -(c(\nabla u, \vartheta, q)-c(0,0,0)) \vartheta_{t}-(g(\nabla u, \vartheta, q)-g(0,0,0)) \operatorname{div} q \\
& -\operatorname{tr}\left((B(\nabla u, \vartheta, q)-B(0,0,0)) \nabla u_{t}\right), \\
H:= & -(\tau(\nabla u, \vartheta)-\tau(0,0)) q_{t}-(K(\nabla u, \vartheta)-K(0,0)) \nabla \vartheta .
\end{aligned}
$$

Finally, we introduce for $j \in \mathbb{N}_{0}$ according to (1.5) the notation

$$
u^{j}:=\partial_{t}^{j} u(0), \quad \vartheta^{j}:=\partial_{t}^{j} \vartheta(0) .
$$

Already in the parabolic case it has been proved that curl-free data give the exponential stability; cf. 9. Therefore, we will now turn over to the radially symmetric case which ensures the rotation will vanish. Thus we will succeed for the first time in proving the existence of global solutions of the nonlinear initial boundary value problem (1.4)-(1.6) in hyperbolic thermoelasticity.

But carrying over the results in 9 directly to the multidimensional case, which seemed to be the obvious method, turned out to be too difficult. To overcome these difficulties we will use an appropriate combination of the techniques presented in [12] and [9, respectively.

2. Radial symmetry and local well-posedness. To begin with, we will characterize the term of radial symmetry:

DeFinition 2.1. Let $\Omega \subset \mathbb{R}^{n}$ be a radially symmetric domain, i. e. for all $x \in \Omega$ and $R \in O(n)$, the orthogonal group of $\mathbb{R}^{n}$, we get $R x \in \Omega$.

(i) A function $f: \Omega \longrightarrow \mathbb{R}$ is called radially symmetric, if for all $R \in O(n)$ it holds

$$
f \circ R=f .
$$

(ii) A vector field $v: \Omega \longrightarrow \mathbb{R}^{n}$ is called radially symmetric, if for all $R \in O(n)$ it holds

$$
R^{T} \circ v \circ R=v .
$$

(iii) Let $F$ and $V$ be a set of functions and vector fields, respectively. Then

$$
\begin{aligned}
\stackrel{\circ}{F} & :=\{f \in F: f \text { radially symmetric }\}, \\
\stackrel{\circ}{V} & :=\{v \in V: v \text { radially symmetric }\} .
\end{aligned}
$$


A subset $\Omega \subset \mathbb{R}^{n}$ is obviously radially symmetric, if and only if the appropriate characteristic function $\chi_{\Omega}: \mathbb{R}^{n} \longrightarrow\{0,1\}$ is. We get the following characterization (folklore, cf. [8], p. 64):

LEMmA 2.2. Let $I:=\{|x|: x \in \Omega\}$.

(i) A function $f: \Omega \longrightarrow \mathbb{R}$ is radially symmetric if and only if there is a function $\varphi_{f}: I \longrightarrow \mathbb{R}$ with $f(x)=\varphi_{f}(|x|)$ for all $x \in \Omega$.

(ii) A vector field $v: \Omega \longrightarrow \mathbb{R}^{n}$ is radially symmetric if and only if $v(0)=0$ (provided that $0 \in \Omega$ ) and there is a function $\varphi_{v}: I \longrightarrow \mathbb{R}$ with $v(x)=\varphi_{v}(|x|) \frac{x}{|x|}$ for all $x \in \Omega \backslash\{0\}$.

Proof. (i) Take $v \in \mathbb{R}^{n}$ with $|v|=1$. The function $\varphi_{f}: I \longrightarrow \mathbb{R}$ is now declared by

$$
\varphi_{f}(r):=f(r v) .
$$

For $x \in \Omega$ there is $R \in O(n)$ with $R x=|x| v$, and we get, as asserted,

$$
f(x)=f(R x)=f(|x| v)=\varphi_{f}(|x|) .
$$

(ii) Let $x \in \Omega \backslash\{0\}$. Then choose $x^{\perp} \in \Omega$ with $x \cdot x^{\perp}=0$, and $R \in O(n)$ with $R x=x$ and $R x^{\perp}=-x^{\perp}$. It follows that

$$
v(x) \cdot x^{\perp}=R^{T} v(R x) \cdot x^{\perp}=v(R x) \cdot R x^{\perp}=-v(x) \cdot x^{\perp},
$$

hence $v(x) x^{\perp}=0$. Since $x^{\perp}$ is an arbitrary element of the orthogonal complement of $\operatorname{lin}_{\mathbb{R}}\{x\}$, a function $f: \Omega \longrightarrow \mathbb{R}$ with

$$
v(x)=f(x) \frac{x}{|x|}
$$

for $x \neq 0$ must exist. Thus $f(x)=v(x) \cdot \frac{x}{|x|}$ holds, and for all $R \in O(n)$ we get

$$
f(R x)=v(R x) \cdot \frac{R x}{|x|}=R^{T} v(R x) \cdot \frac{x}{|x|}=v(x) \cdot \frac{x}{|x|}=f(x) .
$$

For this reason $f$ is a radially symmetric function, and according to (i) we have $f(x)=\varphi_{v}(|x|)$.

Obviously, the inversion holds in both cases.

To develop a theory of radially symmetric solutions of (1.4), the appearing coefficients must transform as follows:

Assumption 2.3. For all $R \in O(n), \zeta \in \mathbb{R}^{n \times n}, \eta \in \mathbb{R}$, and $\chi \in \mathbb{R}^{n}$, we have

$$
M\left(R^{T} \zeta R, \eta, R^{T} \chi\right)=R^{T} M(\zeta, \eta, \chi) R \quad \text { for } \quad M \in\{A, B\}
$$

and

$$
f\left(R^{T} \zeta R, \eta, R^{T} \chi\right)=f(\zeta, \eta, \chi) \quad \text { for } \quad f \in\{c, g\}
$$

as well as

$$
N\left(R^{T} \zeta R, \eta\right)=R^{T} N(\zeta, \eta) R \quad \text { for } \quad N \in\{T, K\}
$$


This guarantees that the operators in (1.4) preserve the radial symmetry. For example, let $v:=A(\nabla u, \vartheta, q) \Delta u$. If $u, \vartheta$, and $q$ are radially symmetric, then so is $v$ :

$$
\begin{aligned}
R^{T} v(R x) & =R^{T} A((\nabla u)(R x), \vartheta(R x), q(R x)) R R^{T}(\Delta u)(R x) \\
& =A\left(R^{T}(\nabla u)(R x) R, \vartheta(R x), R^{T} q(R x)\right) R^{T}(\Delta u)(R x) \\
& =A(\nabla u(x), \vartheta(x), q(x)) \Delta u(x) \\
& =v(x) .
\end{aligned}
$$

Before formulating the local well-posedness theorem, we recall the following SoBOLEV embedding theorem which is important for the proof and can be found, for example, in 1]:

TheOREM 2.4. Let $\Omega \subset \mathbb{R}^{n}, n \in \mathbb{N}$, be a domain satisfying the cone condition. For $s \in \mathbb{N}_{0}$ with $s \geq[n / 2]+1$ the following embeddings hold:

$$
H^{s}(\Omega, \mathbb{R}) \hookrightarrow C_{b}^{0}(\Omega, \mathbb{R}) \quad \text { and } \quad H^{s}\left(\Omega, \mathbb{R}^{n}\right) \hookrightarrow C_{b}^{0}\left(\Omega, \mathbb{R}^{n}\right)
$$

Thus we need at least $s=2$ for $n=2,3$. Even in the case of radial symmetry this cannot be improved in general. But if we consider a spherical shell, then we can deduce from Lemma 2.2 and the embedding theorem in one space dimension the following proposition for radially symmetric functions and vector fields, respectively. It says that we get the same embedding theorem for a spherical shell in higher space dimensions as for a bounded interval:

LEMma 2.5 (Embedding theorem for the spherical shell). For the spherical shell $S:=$ $B(0, r, R):=\left\{x \in \mathbb{R}^{n}: r<|x|<R\right\}, 0<r<R$, we get

$$
\stackrel{\circ}{H}^{1}(S, \mathbb{R}) \hookrightarrow \stackrel{\circ}{C}_{b}^{0}(S, \mathbb{R}) \text { and } \stackrel{\circ}{H}^{1}\left(S, \mathbb{R}^{n}\right) \hookrightarrow \stackrel{\circ}{C}_{b}^{0}\left(S, \mathbb{R}^{n}\right)
$$

Using spherical coordinates we can now transform the multidimensional, radially symmetric problem to a one-dimensional one with additionally local dependent coefficients. Hence, we can directly transfer the local well-posedness theorem, which is given for $n=1$ in [11, to the case of a spherical shell. Note that due to the radial symmetry (the existence of $\operatorname{div} q$, for example) immediately gives the optimal regularity $q \in H^{1}\left(S, \mathbb{R}^{n}\right)$.

Theorem 2.6. Let $s \geq 3$ and $S:=B(0, r, R) \subset \mathbb{R}^{n}$ with $0<r<R, n \in \mathbb{N}$. Assume the following compatibility conditions:

$$
\begin{aligned}
& u^{k} \in \stackrel{\circ}{H}^{s-k}\left(S, \mathbb{R}^{n}\right) \cap \stackrel{\circ}{H}_{0}^{1}\left(S, \mathbb{R}^{n}\right) \quad \text { for } \quad k=0,1, \ldots, s-1 \quad \text { and } \quad u^{s} \in \stackrel{\circ}{L}^{2}\left(S, \mathbb{R}^{n}\right), \\
& \vartheta^{l} \in \stackrel{\circ}{H}^{s-1-l}(S, \mathbb{R}) \cap \stackrel{\circ}{H}_{0}^{1}(S, \mathbb{R}) \quad \text { for } \quad l=0, \ldots, s-2 \quad \text { and } \quad \vartheta^{s-1} \in \stackrel{\circ}{L}^{2}(S, \mathbb{R}),
\end{aligned}
$$


as well as $q^{0} \in \stackrel{\circ}{H}^{s-1}\left(S, \mathbb{R}^{n}\right)$. Then, for sufficiently small $T>0$, the initial boundary value problem (1.4), (1.5), (1.6) has a unique solution $(u, \vartheta, q)$ on $[0, T]$ with

$$
\begin{array}{ll}
u \in \bigcap_{k=0}^{s-1} C^{k}\left([0, T], \stackrel{\circ}{H}^{s-k}\left(S, \mathbb{R}^{n}\right) \cap \stackrel{\circ}{H}_{0}^{1}\left(S, \mathbb{R}^{n}\right)\right), & \partial_{t}^{s} u \in C^{0}\left([0, T], \stackrel{\circ}{L}^{2}\left(S, \mathbb{R}^{n}\right)\right), \\
\vartheta \in \bigcap_{l=0}^{s-2} C^{l}\left([0, T], \stackrel{\circ}{H}^{s-1-l}(K, \mathbb{R}) \cap \stackrel{\circ}{H_{0}^{1}}(S, \mathbb{R})\right), & \partial_{t}^{s-1} \vartheta \in C^{0}\left([0, T], \stackrel{\circ}{L}^{2}(S, \mathbb{R})\right), \\
q \in \bigcap_{m=0}^{s-2} C^{m}\left([0, T], \stackrel{\circ}{H}^{s-1-m}\left(S, \mathbb{R}^{n}\right)\right), & \partial_{t}^{s-1} q \in C^{0}\left([0, T], \stackrel{\circ}{L}^{2}\left(S, \mathbb{R}^{n}\right)\right) .
\end{array}
$$

In addition to the one derivative from Proposition [2.5, two more are required to obtain a local existence result. If we don't want to restrict ourselves to spherical shells we may formulate for arbitrary, radially symmetric domains - in particular for a ball - the following theorem of local well-posedness:

Theorem 2.7. Let $s \geq[n / 2]+3$. Furthermore, assume that the compatibility conditions in Theorem 2.6 hold. Then, for sufficiently small $T>0$, the initial boundary value problem (1.4), (1.5), (1.6) has a unique solution $(u, \vartheta, q)$ on $[0, T]$ with

$u \in \bigcap_{k=0}^{s} C^{k}\left([0, T], \stackrel{\circ}{H}^{s-k}\left(\Omega, \mathbb{R}^{n}\right)\right), \quad(\vartheta, q) \in \bigcap_{l=0}^{s-1} C^{l}\left([0, T], \stackrel{\circ}{H}^{s-1-l}(\Omega, \mathbb{R}) \times \stackrel{\circ}{H}^{s-1-l}\left(\Omega, \mathbb{R}^{n}\right)\right)$.

Thus the treatment of the two- or three-dimensional case requires that, at least, $s=4$. For the expansion to a global existence theorem of small solutions we will therefore take all the derivatives up to order four into account. Before beginning this we still need one more preliminary lemma.

In analogy to Lemma 2.3 from [9] we verify the following

Lemma 2.8. Let $v$ with $v_{i \mid j}=v_{j \mid i}$ solve the equation of elasticity

$$
\begin{aligned}
v_{i|t| t}-A_{i j} v_{j|k| k}=h_{i} & \text { in } \quad[0, \infty) \times \Omega, \\
\left.v\right|_{\partial \Omega}=0 & \text { for } \quad t \geq 0
\end{aligned}
$$

with $A=A(t, x), h=h(t, x)$. Then we get

$$
\begin{aligned}
\oint_{\partial \Omega} A_{i j} v_{j \mid l} v_{i \mid l} \mathrm{~d} S= & 2 \frac{\mathrm{d}}{\mathrm{d} t} \int_{\Omega} v_{i \mid t} \sigma_{k} v_{i \mid k} \mathrm{~d} x+\int_{\Omega} v_{i \mid t} v_{i \mid t} \sigma_{k \mid k} \mathrm{~d} x+2 \int_{\Omega} A_{i j} v_{j \mid l} \sigma_{k \mid l} v_{i \mid k} \mathrm{~d} x \\
& -\int_{\Omega} A_{i j} v_{j \mid l} v_{i \mid l} \sigma_{k \mid k} \mathrm{~d} x-2 \int_{\Omega} h_{i} \sigma_{k} v_{i \mid k} \mathrm{~d} x+R,
\end{aligned}
$$

where $\sigma \in C^{1}\left(\bar{\Omega}, \mathbb{R}^{n}\right)$ with $\left.\sigma\right|_{\partial \Omega}=\nu$ is a smooth continuation of the normal into the interior, and

$$
R:=2 \int_{\Omega} A_{i j \mid l} v_{j \mid l} \sigma_{k} v_{i \mid k} \mathrm{~d} x-\int_{\Omega} A_{i j \mid k} v_{j \mid l} \sigma_{k} v_{i \mid l} \mathrm{~d} x .
$$

Proof. Multiplying the equation by $\sigma_{k} v_{i \mid k}$ and integrating, we obtain

$$
\int_{\Omega} v_{i|t| t} \sigma_{k} v_{i \mid k} \mathrm{~d} x-\int_{\Omega} A_{i j} v_{i|l| l} \sigma_{k} v_{i \mid k} \mathrm{~d} x=\int_{\Omega} h_{i} \sigma_{k} v_{i \mid k} \mathrm{~d} x .
$$


The first term can be written as

$$
\int_{\Omega} v_{i|t| t} \sigma_{k} v_{i \mid k} \mathrm{~d} x=\frac{\mathrm{d}}{\mathrm{d} t} \int_{\Omega} v_{i \mid t} \sigma_{k} v_{i \mid k} \mathrm{~d} x+\frac{1}{2} \int_{\Omega} v_{i \mid t} v_{i \mid t} \sigma_{k \mid k} \mathrm{~d} x
$$

For the second integral we get

$$
\begin{aligned}
\int_{\Omega} A_{i j} v_{j|l| l} \sigma_{k} v_{i \mid k} \mathrm{~d} x= & \oint_{\partial \Omega} A_{i j} v_{j \mid l} \sigma_{k} \sigma_{l} v_{i \mid k} \mathrm{~d} S-\int_{\Omega} A_{i j} v_{j \mid l} \sigma_{k \mid l} v_{i \mid k} \mathrm{~d} x \\
& -\int_{\Omega} A_{i j} v_{j \mid l} \sigma_{k} v_{i|l| k} \mathrm{~d} x-\int_{\Omega} A_{i j \mid l} v_{j \mid l} \sigma_{k} v_{i \mid k} \mathrm{~d} x
\end{aligned}
$$

and after another partial integration it follows that

$$
\begin{aligned}
\int_{\Omega} A_{i j} v_{j|l| l} \sigma_{k} v_{i \mid k} \mathrm{~d} x= & \oint_{\partial \Omega} A_{i j} v_{j \mid l} \sigma_{k} \sigma_{l} v_{i \mid k} \mathrm{~d} S-\int_{\Omega} A_{i j} v_{j \mid l} \sigma_{k \mid l} v_{i \mid k} \mathrm{~d} x-\oint_{\partial \Omega} A_{i j} v_{j \mid l} \sigma_{k} \sigma_{k} v_{i \mid l} \mathrm{~d} S \\
& +\int_{\Omega} A_{i j} v_{j|l| k} \sigma_{k} v_{i \mid l} \mathrm{~d} x+\int_{\Omega} A_{i j} v_{j \mid l} \sigma_{k \mid k} v_{i \mid l} \mathrm{~d} x \\
& -\int_{\Omega} A_{i j \mid l} v_{j \mid l} \sigma_{k} v_{i \mid k} \mathrm{~d} x+\int_{\Omega} A_{i j \mid k} v_{j \mid l} \sigma_{k} v_{i \mid l} \mathrm{~d} x
\end{aligned}
$$

Due to the assumed symmetry of $A$,

$$
A_{i j} v_{j|l| k} \sigma_{k} v_{i \mid l}=A_{j i} v_{i \mid l} \sigma_{k} v_{j|l| k}=A_{i j} v_{j \mid l} \sigma_{k} v_{i|l| k}
$$

holds. Note that, for the last equality, the indices of summation have been renamed. Therefore we obtain from (2.6) and (2.7)

$$
\begin{aligned}
\int_{\Omega} A_{i j} v_{j|l| l} \sigma_{k} v_{i \mid k} \mathrm{~d} x= & 2 \oint_{\partial \Omega} A_{i j} v_{j \mid l} \sigma_{k} \sigma_{l} v_{i \mid k} \mathrm{~d} S-\oint_{\partial \Omega} A_{i j} v_{j \mid l} v_{i \mid l} \mathrm{~d} S-\int_{\Omega} A_{i j} v_{j|l| l} \sigma_{k} v_{i \mid k} \mathrm{~d} x \\
& -2 \int_{\Omega} A_{i j} v_{j \mid l} \sigma_{k \mid l} v_{i \mid k} \mathrm{~d} x+\int_{\Omega} A_{i j} v_{j \mid l} \sigma_{k \mid k} v_{i \mid l} \mathrm{~d} x-R
\end{aligned}
$$

From $\left.v\right|_{\partial \Omega}=0$ we conclude that

$$
\sigma_{k} \sigma_{l} v_{i \mid k}=\sigma_{k} \sigma_{k} v_{i \mid l}=v_{i \mid l} .
$$

The combination of (2.4), (2.5), (2.8), and (2.9) yields the assertion.

3. Global existence and exponential stability. In the following, we assume $\Omega$ and the initial data to be radially symmetric. Let (2.1), (2.2), and (2.3) be satisfied. Furthermore, we make the following assumption:

Assumption 3.1. For all $\zeta \in \mathbb{R}^{n \times n}$ and $\eta \in \mathbb{R}$, it holds that

$$
K(\zeta, \eta) T(\zeta, \eta)=T(\zeta, \eta) K(\zeta, \eta) .
$$

Note that in the standard case, i. e. $T_{i j}=\tau \delta_{i j}$, this does not mean any restriction.

Now we can prove that, for sufficiently small initial data, the local, radially symmetric solution according to Theorem 2.7 is a global one:

Theorem 3.2. There is an $\varepsilon>0$ such that if

$$
\left\|u^{0}\right\|_{H^{4}}^{2}+\left\|u^{1}\right\|_{H^{3}}^{2}+\left\|\vartheta^{0}\right\|_{H^{3}}^{2}+\left\|q^{0}\right\|_{H^{3}}^{2}<\varepsilon,
$$


then (1.4) has an unique solution

$$
u \in \bigcap_{k=0}^{4} C^{k}\left([0, \infty), \stackrel{\circ}{H}^{4-k}\left(\Omega, \mathbb{R}^{n}\right)\right), \quad(\vartheta, q) \in \bigcap_{l=0}^{3} C^{l}\left([0, \infty), \stackrel{\circ}{H}^{3-l}(\Omega, \mathbb{R}) \times \stackrel{\circ}{H}^{3-l}\left(\Omega, \mathbb{R}^{n}\right)\right) .
$$

Moreover, the system is exponentially stable, i. e. there are constants $C, d>0$ such that for all $t \geq 0$

$$
\Lambda(t):=\sum_{k=0}^{4}\left\|\left(\partial_{t}, \nabla\right)^{k} u(t, \cdot)\right\|^{2}+\sum_{l=0}^{3}\left\|\left(\partial_{t}, \nabla\right)^{l}(\vartheta, q)(t, \cdot)\right\|^{2} \leq C e^{-d t} \Lambda(0) .
$$

Proof. The following proof combines the techniques from [11] and [12]. According to the first paper we will use nonlinear multipliers, while the structure is more similar to that in the latter work. In essence, we will deduce an a priori estimate which simultaneously gives a uniform bound on the highest norms of the local solution allowing a continuationargument and shows the exponential decay.

First we define

$$
\begin{aligned}
E_{1}(t) & :=E[u, \vartheta, q](t) \\
& :=\frac{1}{2} \int_{\Omega}\left(u_{i \mid t} u_{i \mid t}+u_{i \mid k} A_{k l} u_{l \mid i}+c \vartheta^{2}+q_{i} K_{i l}^{\#} T_{l m} q_{m}\right)(t, x) \mathrm{d} x, \\
E_{2}(t) & :=E\left[u_{\mid t}, \vartheta_{\mid t}, q_{\mid t}\right](t)
\end{aligned}
$$

with $K^{\#}:=g K^{-1}$, as well as

$$
E(t):=E_{1}(t)+E_{2}(t) .
$$

According to the assumptions, $K^{\#} T$ is symmetric and positive definite, and from the equations (1.4)-(1.6),

$$
\frac{\mathrm{d}}{\mathrm{d} t} E_{1}=-\int_{\Omega} q_{i} K_{i l}^{\#} q_{l} \mathrm{~d} x+R_{\mathrm{c}}
$$

follows, where

$$
\begin{aligned}
R_{\mathrm{c}}=\int_{\Omega} & \left(\frac{1}{2}\left(u_{i \mid k} A_{k l \mid t} u_{l \mid i}+c_{\mid t} \vartheta^{2}+q_{i}\left(K_{i l}^{\#} T_{l m}\right)_{\mid t} q_{m}\right)\right. \\
& \left.-u_{i \mid k} A_{k l \mid i} u_{l \mid t}+\vartheta B_{i k \mid k} u_{i \mid t}+\vartheta g_{\mid i} q_{i}\right) \mathrm{d} x .
\end{aligned}
$$

The nonlinear term $R_{\mathrm{c}}$ contains only summands of at least third order in $\sqrt{\Lambda}$ as we will prove in the subsequent section. In the following we will refer to all these arising perturbations of cubic type as $R_{\mathrm{c}}$.

Differentiating (1.4) with respect to $t$, we obtain

$$
\frac{\mathrm{d}}{\mathrm{d} t} E_{2}=-\int_{\Omega} q_{i \mid t} K_{i l}^{\#} q_{l \mid t} \mathrm{~d} x+R_{\mathrm{c}} .
$$

The equation (1.4c) gives

$$
\|\nabla \vartheta\|^{2} \leq C_{0}\left(\|q\|^{2}+\left\|q_{\mid t}\right\|^{2}\right)
$$

where $C_{0}$-as well as $C_{i}, i \in \mathbb{N}$, throughout the rest of the proof-is a positive constant independent of $(u, \vartheta, q)$. 
We multiply (1.8a) by $\frac{1}{\alpha} u_{i|k| k}=\frac{1}{\alpha} u_{k|k| i}$ and get, after integration,

$$
\begin{aligned}
\|\Delta u\|^{2} & =\int_{\Omega}\left(\frac{1}{\alpha} u_{i|t| t} u_{k|k| i}+\frac{\beta}{\alpha} \vartheta_{\mid k} u_{i|j| j}\right) \mathrm{d} x+R_{\mathrm{c}} \\
& \leq \int_{\Omega}-\frac{1}{\alpha} u_{i|i| t \mid t} u_{k \mid k} \mathrm{~d} x+\frac{1}{3}\|\Delta u\|^{2}+\frac{3}{4} \frac{\beta^{2}}{\alpha^{2}}\|\nabla \vartheta\|^{2}+R_{\mathrm{c}} \\
& \leq \int_{\Omega}\left(\left(-\frac{1}{\alpha} u_{i|i| t} u_{k \mid k}\right)_{\mid t}+\frac{1}{\alpha} u_{i|i| t} u_{k|k| t}\right) \mathrm{d} x+\frac{1}{3}\|\Delta u\|^{2}+\frac{3}{4} \frac{\beta^{2}}{\alpha^{2}}\|\nabla \vartheta\|^{2}+R_{\mathrm{c}}
\end{aligned}
$$

hence

$$
\frac{2}{3}\|\Delta u\|^{2}+\frac{\mathrm{d}}{\mathrm{d} t} \int_{\Omega} \frac{1}{\alpha} \operatorname{div} u_{\mid t} \operatorname{div} u \mathrm{~d} x \leq \int_{\Omega} \frac{1}{\alpha}\left(\operatorname{div} u_{\mid t}\right)^{2} \mathrm{~d} x+\frac{3}{4} \frac{\beta^{2}}{\alpha^{2}}\|\nabla \vartheta\|^{2}+R_{\mathrm{c}} .
$$

Multiplication of $(1.8 \mathrm{~b})$ by $\frac{3}{\alpha \beta} u_{k|k| t}$ yields

$$
\begin{aligned}
\int_{\Omega} \frac{3}{\alpha}\left(\operatorname{div} u_{\mid t}\right)^{2} \mathrm{~d} x= & -\int_{\Omega}\left(\frac{3 \gamma}{\alpha \beta} q_{i \mid i} u_{k|k| t}+\frac{3 \zeta}{\alpha \beta} \vartheta_{\mid t} u_{k|k| t}\right) \mathrm{d} x+R_{\mathrm{c}} \\
= & \int_{\Omega} \frac{3 \gamma}{\alpha \beta} q_{i} u_{i|k| k \mid t} \mathrm{~d} x-\oint_{\partial \Omega} \frac{3 \gamma}{\alpha \beta} \nu_{i} q_{i} u_{k|k| t} \mathrm{~d} S+\int_{\Omega} \frac{3 \zeta}{\alpha \beta} \vartheta_{|k| t} u_{k \mid t} \mathrm{~d} x+R_{\mathrm{c}} \\
= & \int_{\Omega}\left(\left(\frac{3 \gamma}{\alpha \beta} q_{i} u_{i|k| k}\right)_{\mid t}-\frac{3 \gamma}{\alpha \beta} q_{i \mid t} u_{i|k| k}\right) \mathrm{d} x \\
& +\int_{\Omega}\left(\left(\frac{3 \zeta}{\alpha \beta} \vartheta_{\mid k} u_{k \mid t}\right)_{\mid t}-\frac{3 \zeta}{\alpha \beta} \vartheta_{\mid k} u_{k|t| t}\right) \mathrm{d} x-\oint_{\partial \Omega} \frac{3 \gamma}{\alpha \beta} \nu_{i} q_{i} u_{k|k| t} \mathrm{~d} S+R_{\mathrm{c}}
\end{aligned}
$$

and thus from (1.4a) it follows that

$$
\begin{aligned}
\int_{\Omega} \frac{3}{\alpha}\left(\operatorname{div} u_{\mid t}\right)^{2} \mathrm{~d} x \leq & -\frac{\mathrm{d}}{\mathrm{d} t} G_{1}+\frac{1}{6}\|\Delta u\|^{2}+C_{1}\left\|q_{\mid t}\right\|^{2}+C_{2}\|\nabla \vartheta\|^{2} \\
& -\oint_{\partial \Omega} \frac{3 \gamma}{\alpha \beta} \nu_{i} q_{i} u_{k|k| t} \mathrm{~d} S+R_{\mathrm{c}}
\end{aligned}
$$

with the energy functional

$$
G_{1}(t):=-\int_{\Omega}\left(\frac{3 \gamma}{\alpha \beta} q_{i} u_{i|k| k}+\frac{3 \zeta}{\alpha \beta} \vartheta_{\mid k} u_{k \mid t}\right)(t, x) \mathrm{d} x .
$$

From (3.9) and (3.10) we deduce that

$$
\begin{aligned}
\int_{\Omega} \frac{2}{\alpha}\left(\operatorname{div} u_{\mid t}\right)^{2} \mathrm{~d} x+\frac{1}{2}\|\Delta u\|^{2}+\frac{\mathrm{d}}{\mathrm{d} t} G_{2} \\
\leq C_{3}\|\nabla \vartheta\|^{2}+C_{4}\left\|q_{|t|}\right\|^{2}-\oint_{\partial \Omega} \frac{3 \gamma}{\alpha \beta} \nu_{i} q_{i} u_{k|k| t} \mathrm{~d} S+R_{\mathrm{c}}
\end{aligned}
$$

with

$$
G_{2}(t):=G_{1}(t)+\int_{\Omega} \frac{1}{\alpha} u_{i|i| t} u_{k \mid k}(t, x) \mathrm{d} x .
$$

PoINCARÉ's inequality for $u_{\mid t}$ and $\vartheta$, as well as (1.4a), yield

$$
\left\|u_{|t| t}\right\|^{2}+\left\|u_{\mid t}\right\|^{2}+\|\vartheta\|^{2} \leq C_{5}\left(\|\Delta u\|^{2}+\|\nabla \vartheta\|^{2}+\left\|\nabla u_{\mid t}\right\|^{2}\right) .
$$


From (1.8a) follows (multiply by $u$ )

$$
-\int_{\Omega} \alpha \Delta u \cdot u \mathrm{~d} x=-\int_{\Omega} u_{|t| t} \cdot u \mathrm{~d} x+\int_{\Omega} \beta \nabla \vartheta \cdot u \mathrm{~d} x+R_{\mathrm{c}}
$$

and hence Poincaré's inequality for $u$ yields

$$
\frac{1}{2} \int_{\Omega} \alpha|\nabla u|^{2} \mathrm{~d} x \leq C_{6}\left(\left\|u_{|t| t}\right\|^{2}+\|\nabla \vartheta\|^{2}\right)+R_{\mathrm{c}}
$$

Multiplying (1.8b) by $\frac{1}{\zeta} \vartheta_{\mid t}$, we can easily see that

$$
\begin{aligned}
\left\|\vartheta_{\mid t}\right\|^{2} & \leq \int_{\Omega} \frac{\gamma}{\zeta} q_{i} \vartheta_{|i| t} \mathrm{~d} x+C_{7}\left\|\operatorname{div} u_{\mid t}\right\|^{2}+\frac{1}{2}\left\|\vartheta_{|t|}\right\|^{2}+R_{\mathrm{c}} \\
& =\int_{\Omega}\left(\left(\frac{\gamma}{\zeta} q_{i} \vartheta_{\mid i}\right)_{\mid t}-\frac{\gamma}{\zeta} q_{i \mid t} \vartheta_{\mid i}\right) \mathrm{d} x+C_{8}\left\|\operatorname{div} u_{\mid t}\right\|^{2}+\frac{1}{2}\left\|\vartheta_{\mid t}\right\|^{2}+R_{\mathrm{c}}
\end{aligned}
$$

and for arbitrary $\varepsilon_{1}>0$ we get

$$
\varepsilon_{1}\left\|\vartheta_{\mid t}\right\|^{2}-2 \varepsilon_{1} \frac{\mathrm{d}}{\mathrm{d} t} \int_{\Omega} \frac{\gamma}{\zeta} q_{i} \vartheta_{\mid i} \mathrm{~d} x \leq \varepsilon_{1} C_{9}\left\|q_{\mid t}\right\|^{2}+\varepsilon_{1} C_{10}\|\nabla \vartheta\|^{2}+\varepsilon_{1} C_{11}\left\|\operatorname{div} u_{\mid t}\right\|^{2}+R_{\mathrm{c}}
$$

For some $\varepsilon_{2}>0$, the boundary term appearing in (3.11) can be estimated as follows:

$$
\left|\oint_{\partial \Omega} \frac{3 \gamma}{\alpha \beta} \nu_{i} q_{i} u_{k|k| t} \mathrm{~d} S\right| \leq \frac{C_{12}}{\varepsilon_{2}}\|\nu \cdot q\|_{\partial \Omega}^{2}+\varepsilon_{2}\left\|\nabla u_{\mid t}\right\|_{\partial \Omega}^{2}
$$

Now let $\sigma \in C^{1}\left(\bar{\Omega}, \mathbb{R}^{n}\right)$ be a smooth continuation of the normal into the interior (cf. Lemma 2.8). Then the multiplication of (1.4b) by $\sigma_{k} \vartheta_{|k| t}$ gives

$$
\begin{aligned}
0= & -\int_{\Omega} c \vartheta_{\mid t} \sigma_{k} \vartheta_{|k| t} \mathrm{~d} x-\int_{\Omega} g q_{i \mid i} \sigma_{k} \vartheta_{|k| t} \mathrm{~d} x-\int_{\Omega} B_{i j} u_{i|j| t} \sigma_{k} \vartheta_{|k| t} \mathrm{~d} x \\
= & -\frac{1}{2} \oint_{\partial \Omega} c \vartheta_{\mid t} \vartheta_{\mid t} \mathrm{~d} S+\frac{1}{2} \int_{\Omega} c \vartheta_{\mid t} \sigma_{k \mid k} \vartheta_{\mid t} \mathrm{~d} x-\frac{\mathrm{d}}{\mathrm{d} t} \int_{\Omega} g q_{i \mid i} \sigma_{k} \vartheta_{\mid k} \mathrm{~d} x \\
& +\int_{\Omega} g q_{i|t| i} \sigma_{k} \vartheta_{\mid k} \mathrm{~d} x-\int_{\Omega} B_{i j} u_{i|j| t} \sigma_{k} \vartheta_{|k| t} \mathrm{~d} x+R_{\mathrm{c}} .
\end{aligned}
$$

Thus, with (1.4c),

$$
\begin{aligned}
0= & -\frac{1}{2} \oint_{\partial \Omega} c \vartheta_{\mid t} \vartheta_{\mid t} \mathrm{~d} S+\frac{1}{2} \int_{\Omega} c \vartheta_{\mid t} \sigma_{k \mid k} \vartheta_{\mid t} \mathrm{~d} x-\frac{\mathrm{d}}{\mathrm{d} t} \int_{\Omega} g q_{i \mid i} \sigma_{k} \vartheta_{\mid k} \mathrm{~d} x \\
& -\int_{\Omega} g T_{i j}^{-1} q_{j \mid i} \sigma_{k} \vartheta_{\mid k} \mathrm{~d} x-\int_{\Omega} g T_{i j}^{-1} K_{j l} \vartheta_{|l| i} \sigma_{k} \vartheta_{\mid k} \mathrm{~d} x-\int_{\Omega} B_{i j} u_{i|j| t} \sigma_{k} \vartheta_{|k| t} \mathrm{~d} x+R_{\mathrm{c}} .
\end{aligned}
$$


Because we have $\sigma_{i} \vartheta_{\mid k}=\sigma_{k} \vartheta_{\mid i}$ on $\partial \Omega$ as a result of the radial symmetry of $\vartheta$, and due to the symmetry of $T^{-1} K$, the following equality holds:

$$
\begin{aligned}
\int_{\Omega} g T_{i j}^{-1} K_{j l} \vartheta_{|l| i} \sigma_{k} \vartheta_{\mid k} \mathrm{~d} x= & \oint_{\partial \Omega} g T_{i j}^{-1} K_{j l} \vartheta_{\mid l} \sigma_{i} \sigma_{k} \vartheta_{\mid k} \mathrm{~d} S-\int_{\Omega} g T_{i j}^{-1} K_{j l} \vartheta_{\mid l} \sigma_{k \mid i} \vartheta_{\mid k} \mathrm{~d} x \\
& -\int_{\Omega} g T_{i j}^{-1} K_{j l} \vartheta_{\mid l} \sigma_{k} \vartheta_{|i| k} \mathrm{~d} x+R_{\mathrm{c}} \\
= & \oint_{\partial \Omega} g T_{i j}^{-1} K_{j l} \vartheta_{\mid l} \vartheta_{\mid i} \mathrm{~d} S-\int_{\Omega} g T_{i j}^{-1} K_{j l} \vartheta_{\mid l} \sigma_{k \mid i} \vartheta_{\mid k} \mathrm{~d} x \\
& -\frac{1}{2} \oint_{\partial \Omega} g T_{i j}^{-1} K_{j l} \vartheta_{\mid l} \vartheta_{\mid i} \mathrm{~d} S+\frac{1}{2} \int_{\Omega} g T_{i j}^{-1} K_{j l} \vartheta_{\mid l} \sigma_{k \mid k} \vartheta_{\mid i} \mathrm{~d} x+R_{\mathrm{c}}
\end{aligned}
$$

This gives in combination with (3.18)

$$
\begin{aligned}
0= & \oint_{\partial \Omega} c \vartheta_{\mid t} \vartheta_{\mid t} \mathrm{~d} S-\int_{\Omega} c \vartheta_{\mid t} \sigma_{k \mid k} \vartheta_{\mid t} \mathrm{~d} x+2 \frac{\mathrm{d}}{\mathrm{d} t} \int_{\Omega} g q_{i \mid i} \sigma_{k} \vartheta_{\mid k} \mathrm{~d} x \\
& +2 \int_{\Omega} g T_{i j}^{-1} q_{j \mid i} \sigma_{k} \vartheta_{\mid k} \mathrm{~d} x+\oint_{\partial \Omega} g T_{i j}^{-1} K_{j l} \vartheta_{\mid l} \vartheta_{\mid i} \mathrm{~d} S-2 \int_{\Omega} g T_{i j}^{-1} K_{j l} \vartheta_{\mid l} \sigma_{k \mid i} \vartheta_{\mid k} \mathrm{~d} x \\
& +\int_{\Omega} g T_{i j}^{-1} K_{j l} \vartheta_{\mid l} \sigma_{k \mid k} \vartheta_{\mid i} \mathrm{~d} x+2 \int_{\Omega} B_{i j} u_{i|j| t} \sigma_{k} \vartheta_{|k| t} \mathrm{~d} x+R_{\mathrm{c}}
\end{aligned}
$$

Defining

$$
\begin{aligned}
v_{i} & :=u_{i \mid t}, \\
h_{i} & :=-B_{i j} \vartheta_{|j| t}-B_{i j \mid t} \vartheta_{\mid j}+A_{i j \mid t} u_{j|l| l},
\end{aligned}
$$

the time-differentiated equality (1.4a) is equivalent to

$$
\begin{aligned}
v_{i|t| t}-A_{i j} v_{j|l| l} & =h_{i}, \\
\left.v\right|_{\partial \Omega} & =0,
\end{aligned}
$$

and Lemma 2.8 yields

$$
\begin{aligned}
\oint_{\partial \Omega} A_{i j} u_{j|l| t} u_{i|l| t} \mathrm{~d} S= & 2 \frac{\mathrm{d}}{\mathrm{d} t} \int_{\Omega} u_{i|t| t} \sigma_{k} u_{i|k| t} \mathrm{~d} x+\int_{\Omega}\left|u_{|t| t \mid}\right|^{2} \sigma_{k \mid k} \mathrm{~d} x+2 \int_{\Omega} A_{i j} u_{j|l| t} \sigma_{k \mid l} u_{i|k| t} \mathrm{~d} x \\
& -\int_{\Omega} A_{i j} u_{j|l| t} \sigma_{k \mid k} u_{i|l| t} \mathrm{~d} x+2 \int_{\Omega} B_{i j} \vartheta_{|j| t} \sigma_{k} u_{i|k| t} \mathrm{~d} x+R_{\mathrm{c}}
\end{aligned}
$$

By partial integration we get

$$
\begin{aligned}
\int_{\Omega} B_{i j} \vartheta_{|j| t} \sigma_{k} u_{i|k| t} \mathrm{~d} x= & -\int_{\Omega} B_{i j} \vartheta_{\mid t} \sigma_{k \mid j} u_{i|k| t} \mathrm{~d} x-\int_{\Omega} B_{i j} \vartheta_{\mid t} \sigma_{k} u_{i|j| k \mid t} \mathrm{~d} x+R_{\mathrm{c}} \\
= & -\int_{\Omega} B_{i j} \vartheta_{\mid t} \sigma_{k \mid j} u_{i|k| t} \mathrm{~d} x+\int_{\Omega} B_{i j} \vartheta_{|k| t} \sigma_{k} u_{i|j| t} \mathrm{~d} x \\
& +\int_{\Omega} B_{i j} \vartheta_{\mid t} \sigma_{k \mid k} u_{i|j| t} \mathrm{~d} x+R_{\mathrm{c}}
\end{aligned}
$$

thus

$$
\int_{\Omega} B_{i j} \vartheta_{|j| t} \sigma_{k} u_{i|k| t} \mathrm{~d} x=\int_{\Omega} B_{i j} u_{i|j| t} \sigma_{k} \vartheta_{|k| t} \mathrm{~d} x+\int_{\Omega} B_{i j} \vartheta_{\mid t}\left(\sigma_{k \mid k} u_{i|j| t}-\sigma_{k \mid j} u_{i|k| t}\right) \mathrm{d} x .
$$


From (1.7) and (3.19)-(3.21) follows

$$
\begin{aligned}
& \oint_{\partial \Omega} c\left(\vartheta_{\mid t}\right)^{2} \mathrm{~d} S+\oint_{\partial \Omega} g T_{i j}^{-1} K_{j l} \vartheta_{\mid l} \vartheta_{\mid i} \mathrm{~d} S+A_{0}\left\|\nabla u_{\mid t}\right\|_{\partial \Omega}^{2} \\
& +\frac{\mathrm{d}}{\mathrm{d} t} \int_{\Omega} 2\left(g q_{i \mid i} \sigma_{k} \vartheta_{\mid k}-u_{i|t| t} \sigma_{k} u_{i|k| t}\right) \mathrm{d} x \\
& \quad \leq C_{13}\left(\|q\|^{2}+\left\|\vartheta_{\mid t}\right\|^{2}+\|\nabla \vartheta\|^{2}+\left\|\nabla u_{\mid t}\right\|^{2}+\|\Delta u\|^{2}\right)+R_{\mathrm{c}} .
\end{aligned}
$$

Thereto one has to note that $(1.4 \mathrm{~b})$ gives

$$
\begin{aligned}
\|\operatorname{div} q\|^{2} & =\left\|g^{-1} c \vartheta_{\mid t}+g^{-1} B \cdot \nabla u_{\mid t}\right\|^{2} \\
& \leq C_{14}\left(\left\|\vartheta_{\mid t}\right\|^{2}+\left\|\nabla u_{|t|}\right\|^{2}\right),
\end{aligned}
$$

and that Theorem 8.6 from [10] can be applied in the case of radial symmetry; i.e., there is a positive constant $C$ with $\|\nabla q\| \leq C(\|\operatorname{div} q\|+\|q\|)$, hence

$$
\left|\int_{\Omega} g T_{i j}^{-1} q_{j \mid i} \sigma_{k} \vartheta_{\mid k} \mathrm{~d} x\right| \leq C_{15}\left(\|q\|^{2}+\left\|\vartheta_{\mid t}\right\|^{2}+\left\|\nabla u_{\mid t}\right\|^{2}+\|\nabla \vartheta\|^{2}\right) .
$$

Multiplication of (1.4b) by $\sigma_{k} q_{k}$ yields

$$
\int_{\Omega} c \vartheta_{\mid t} \sigma_{k} q_{k} \mathrm{~d} x+\int_{\Omega} g q_{i \mid i} \sigma_{k} q_{k} \mathrm{~d} x+\int_{\Omega} B_{i j} u_{i|j| t} \sigma_{k} q_{k} \mathrm{~d} x=0 .
$$

We will treat these three terms separately. For $\varepsilon_{3}>0$ we estimate

$$
\left|\int_{\Omega} c \vartheta_{\mid t} \sigma_{k} q_{k} \mathrm{~d} x\right| \leq \varepsilon_{3}\left\|\vartheta_{\mid t}\right\|^{2}+\frac{C_{16}}{\varepsilon_{3}}\|q\|^{2}
$$

and

$$
\left|\int_{\Omega} B_{i j} u_{i|j| t} \sigma_{k} q_{k} \mathrm{~d} x\right| \leq \varepsilon_{3}\left\|\nabla u_{\mid t}\right\|^{2}+\frac{C_{17}}{\varepsilon_{3}}\|q\|^{2} .
$$

It is easy to verify that

$$
\begin{aligned}
\int_{\Omega} g q_{i \mid i} \sigma_{k} q_{k} \mathrm{~d} x & =\oint_{\partial \Omega} g \sigma_{i} q_{i} \sigma_{k} q_{k} \mathrm{~d} S-\int_{\Omega} g q_{i} \sigma_{k \mid i} q_{k} \mathrm{~d} x-\int_{\Omega} g q_{i} \sigma_{k} q_{i \mid k} \mathrm{~d} x+R_{\mathrm{c}} \\
& =\frac{1}{2} \oint_{\partial \Omega} g q_{i} q_{i} \mathrm{~d} S-\int_{\Omega} g q_{i} \sigma_{k \mid i} q_{k} \mathrm{~d} x+\frac{1}{2} \int_{\Omega} g q_{i} \sigma_{k \mid k} q_{i} \mathrm{~d} x+R_{\mathrm{c}}
\end{aligned}
$$

(note $q_{i} \sigma_{k}=q_{k} \sigma_{i}$ and $q_{k \mid i}=q_{i \mid k}$ because of the symmetry); hence the combination of the equations (1.7) and (3.23)-(3.26) yields

$$
g_{0}\|q\|_{\partial \Omega}^{2} \leq \varepsilon_{3}\left(\left\|\vartheta_{\mid t}\right\|^{2}+\left\|\nabla u_{\mid t}\right\|^{2}\right)+\frac{C_{18}}{\varepsilon_{3}}\|q\|^{2}+R_{\mathrm{c}} .
$$

Finally, for sufficiently small $\varepsilon_{1}, \varepsilon_{2}$, and $\varepsilon_{3}$, we can deduce from (3.8), (3.11), (3.15), (3.16), (3.22), and (3.27) that

$$
\frac{1}{\alpha}\left\|\nabla u_{\mid t}\right\|^{2}+\frac{1}{4}\|\Delta u\|^{2}+C_{19}\left\|\vartheta_{\mid t}\right\|^{2}+\frac{\mathrm{d}}{\mathrm{d} t} H \leq C_{20}\left(\|q\|^{2}+\left\|q_{\mid t}\right\|^{2}\right)+R_{\mathrm{c}}
$$

with

$$
H(t):=G_{2}(t)+\frac{2 \varepsilon_{2}}{A_{0}} \int_{\Omega}\left(g q_{i \mid i} \sigma_{k} \vartheta_{\mid k}-u_{i|t| t} \sigma_{k} u_{i|k| t}\right) \mathrm{d} x-2 \varepsilon_{1} \int_{\Omega} \frac{g}{c} q_{i} \vartheta_{i} \mathrm{~d} x .
$$


An appropriate LYAPUNOV functional is given by

$$
\tilde{\lambda}[u, \vartheta, q](t):=\frac{1}{\varepsilon_{4}} E(t)+H(t)
$$

with some $\varepsilon_{4}>0$, because the combination of (3.7), (3.13), (3.14), and (3.28) yields (choose $\varepsilon_{4}$ sufficiently small)

$$
\frac{\mathrm{d}}{\mathrm{d} t} \tilde{\lambda}[u, \vartheta, q](t) \leq-C_{21} E(t)+R_{\mathrm{c}} .
$$

By construction, $\tilde{\lambda}[u, \vartheta, q]$ and $E$ are equivalent; i.e. for $\varepsilon_{4}$ small enough there are constants $C_{22}$ and $C_{23}$ such that

$$
C_{22} E(t) \leq \tilde{\lambda}[u, \vartheta, q](t) \leq C_{23} E(t)
$$

for all $t \geq 0$. Therefore, (3.30) leads to

$$
\frac{\mathrm{d}}{\mathrm{d} t} \tilde{\lambda}[u, \vartheta, q](t) \leq-C_{24} \tilde{\lambda}[u, \vartheta, q](t)+C_{25} \Lambda^{3 / 2}(t) .
$$

Repeating all the calculations also for higher time derivatives, we get, for $k=1,2$,

$$
\frac{\mathrm{d}}{\mathrm{d} t} \tilde{\lambda}\left[\partial_{t}^{k} u, \partial_{t}^{k} \vartheta, \partial_{t}^{k} q\right](t) \leq-C_{24} \tilde{\lambda}\left[\partial_{t}^{k} u, \partial_{t}^{k} \vartheta, \partial_{t}^{k} q\right](t)+C_{26} \Lambda^{3 / 2}(t),
$$

since all appearing perturbation terms are cubic in $\sqrt{\Lambda}$. Now let

$$
\tilde{\Lambda}(t):=\sum_{k=0}^{2} \tilde{\lambda}\left[\partial_{t}^{k} u, \partial_{t}^{k} \vartheta, \partial_{t}^{k} q\right](t) ;
$$

then

$$
\tilde{\Lambda}(t) \leq C_{27} \Lambda(t) .
$$

Differentiating (1.4) adequately many times with respect to $t$ one can see that there are $C_{28}$ and $C_{29}$ such that

$$
\Lambda(t) \leq C_{28} \tilde{\Lambda}(t)+C_{29} \Lambda^{3 / 2}(t) .
$$

(Thereto one may also consider the argumentation in the following section about the cubic terms.)

Now we make a first a priori assumption:

$$
\Lambda(0)<\left(\frac{1}{2 C_{29}}\right)^{2}
$$

Due to the continuity of $\Lambda$ there is $t_{0}>0$ such that

$$
\Lambda(t) \leq\left(\frac{1}{2 C_{29}}\right)^{2}
$$

for all $t \in\left[0, t_{0}\right]$; hence

$$
C_{29} \Lambda^{3 / 2}(t) \leq \frac{1}{2} \Lambda(t)
$$

and together with (3.35) this gives

$$
\Lambda(t) \leq 2 C_{28} \tilde{\Lambda}(t) .
$$


Noting (3.34) we get the equivalence

$$
\frac{1}{2 C_{28}} \Lambda(t) \leq \tilde{\Lambda}(t) \leq C_{27} \Lambda(t)
$$

for all $t \in\left[0, t_{0}\right]$. All in all, from (3.32), (3.33), and (3.38),

$$
\frac{\mathrm{d}}{\mathrm{d} t} \tilde{\Lambda}(t) \leq-C_{30} \tilde{\Lambda}(t)+C_{31} \tilde{\Lambda}^{3 / 2}(t)
$$

follows for the mentioned time-interval.

Second, we now assume

$$
\tilde{\Lambda}(0)<\left(\frac{C_{30}}{2 C_{31}}\right)^{2}
$$

Then there is $t_{1} \in\left(0, t_{0}\right]$ with

$$
\tilde{\Lambda}(t) \leq\left(\frac{C_{30}}{2 C_{31}}\right)^{2}
$$

for all $t \in\left[0, t_{1}\right]$, and in this interval we conclude from (3.39)

$$
\frac{\mathrm{d}}{\mathrm{d} t} \tilde{\Lambda}(t) \leq-\frac{C_{30}}{2} \tilde{\Lambda}(t)
$$

and so we get the estimate

$$
\tilde{\Lambda}(t) \leq e^{-\frac{C_{30}}{2} t} \tilde{\Lambda}(0) .
$$

In particular, one has, due to (3.37) for $t \in\left[0, t_{1}\right]$,

$$
\Lambda(t) \leq 2 C_{28} e^{-\frac{c_{30}}{2} t} \tilde{\Lambda}(0) .
$$

Let us now make the third a priori assumption:

$$
\tilde{\Lambda}(0)<\frac{1}{4 C_{28}}\left(\frac{1}{2 C_{29}}\right)^{2},
$$

and

$$
\Lambda(t)<\frac{1}{2}\left(\frac{1}{2 C_{29}}\right)^{2}
$$

follows for $t \in\left[0, t_{1}\right]$. Note that since we have (3.38), the condition (3.41) implies (3.36). Now we can proceed at $t=t_{1}$ with the same arguments and finally obtain for all $t \geq 0$ the inequality (3.42). Thus, (3.38) as well as (3.40) hold for all times, and we get for $t \geq 0$

$$
\Lambda(t) \leq 2 C_{27} C_{28} e^{-\frac{C_{30}}{2} t} \Lambda(0)
$$

if $\Lambda(0)<\varepsilon_{0}$, where

$$
\varepsilon_{0}:=\min \left\{\frac{1}{C_{27}}\left(\frac{C_{30}}{2 C_{31}}\right)^{2}, \frac{1}{4 C_{27} C_{28}}\left(\frac{1}{2 C_{29}}\right)^{2}\right\} .
$$

Finally, note that there is $\varepsilon>0$ such that $\Lambda(0)<\varepsilon_{0}$ as far as

$$
\left\|u^{0}\right\|_{H^{4}}^{2}+\left\|u^{1}\right\|_{H^{3}}^{2}+\left\|\vartheta^{0}\right\|_{H^{3}}^{2}+\left\|q^{0}\right\|_{H^{3}}^{2}<\varepsilon .
$$


To conclude the proof it remains to show that the appearing terms $R_{\mathrm{c}}$ are at least cubic.

4. Cubic terms. In addition to the embedding Theorem 2.4 we still need one more, cf. [1]:

TheOREm 4.1. Let $\Omega \subset \mathbb{R}^{n}$ be a domain satisfying the cone property. Then the following embeddings hold:

$$
H^{1}(\Omega, \mathbb{R}) \hookrightarrow L^{4}(\Omega, \mathbb{R}) \quad \text { and } \quad H^{1}\left(\Omega, \mathbb{R}^{n}\right) \hookrightarrow L^{4}\left(\Omega, \mathbb{R}^{n}\right) .
$$

Combining theorems 2.4 and 4.1, we find that there are constants $C_{L^{\infty}}, C_{L^{4}}>0$ such that the local solution $(u, \vartheta, q)$ satisfies

$$
\left\|\partial_{t}^{2} u\right\|_{\infty}+\left\|\partial_{t} u\right\|_{W^{1, \infty}}+\|u\|_{W^{2, \infty}}+\left\|\partial_{t}(\vartheta, q)\right\|_{\infty}+\|(\vartheta, q)\|_{W^{1, \infty}} \leq C_{L^{\infty}} \Lambda^{1 / 2}
$$

and

$$
\begin{aligned}
& \left\|\partial_{t}^{3} u\right\|_{L^{4}}+\left\|\partial_{t}^{2} u\right\|_{W^{1,4}}+\left\|\partial_{t} u\right\|_{W^{2,4}}+\|u\|_{W^{3,4}} \\
& +\left\|\partial_{t}^{2}(\vartheta, q)\right\|_{L^{4}}+\left\|\partial_{t}(\vartheta, q)\right\|_{W^{1,4}}+\|(\vartheta, q)\|_{W^{2,4}} \leq C_{L^{4}} \Lambda^{1 / 2}
\end{aligned}
$$

That the arising perturbations $R_{\mathrm{c}}$ are at least cubic in $\sqrt{\Lambda}$ will be shown with three examples of the most problematic terms. All the others can be treated entirely in the same way.

As mentioned before, a calculation analogous to that presented for $\tilde{\lambda}[u, \vartheta, q]$ gives the equivalent to (3.32) for $\tilde{\lambda}\left[\partial_{t}^{2} u, \partial_{t}^{2} \vartheta, \partial_{t}^{2} q\right]$. In (3.7) then the following summand appears in $R_{\mathrm{c}}$ due to both of the additional time-derivatives:

$$
\begin{aligned}
& \left|\int_{\Omega} u_{i|t| t|t| t}\left(A_{i j|t| t \mid t} \Delta u_{j}+A_{i j|t| t} \Delta u_{j \mid t}+A_{i j \mid t} \Delta u_{j|t| t}\right) \mathrm{d} x\right| \\
\leq & C\left(\Lambda^{1 / 2}\left\|\partial_{t}^{3} A\right\|\left\|\Delta u_{j}\right\|_{\infty}+\Lambda^{1 / 2}\left\|\partial_{t}^{2} A\right\|_{L^{4}}\left\|\partial_{t} \Delta u\right\|_{L^{4}}+\Lambda\left\|\partial_{t} A\right\|_{\infty}\right) \\
\leq & C\left(\Lambda\left\|\partial_{t}^{3}(\nabla u, \vartheta, q)\right\|+\Lambda\left\|\partial_{t}^{2}(\nabla u, \vartheta, q)\right\|\left\|\partial_{t}(\nabla u, \vartheta, q)\right\|_{\infty}+\Lambda\left\|\partial_{t}(\nabla u, \vartheta, q)\right\|_{\infty}^{3}\right. \\
& \left.\quad+\Lambda\left\|\partial_{t}^{2}(\nabla u, \vartheta, q)\right\|_{L^{4}}+\Lambda\left\|\partial_{t}(\nabla u, \vartheta, q)\right\|_{\infty}^{2}+\Lambda\left\|\partial_{t}(\nabla u, \vartheta, q)\right\|_{\infty}\right) \\
\leq & C\left(\Lambda^{3 / 2}+\Lambda^{2}+\Lambda^{5 / 2}\right) .
\end{aligned}
$$

Therefore, $C>0$ is a constant independent of $(u, \vartheta, q)$ which may change from time to time.

Now let

$$
\tilde{F}:=A_{|t| t} \Delta u+2 A_{\mid t} \Delta u_{\mid t}+\left(A-\alpha E_{n}\right) \Delta u_{|t| t}-B_{|t| t} \nabla \vartheta-2 B_{\mid t} \nabla \vartheta_{\mid t}-\left(B-\beta E_{n}\right) \nabla \vartheta_{|t| t} .
$$

Then we have to consider a term similar to

$$
\int_{\Omega} \tilde{F} \Delta u_{|t| t} \mathrm{~d} x
$$

in the derivation of (3.9) for $\tilde{\lambda}\left[\partial_{t}^{2} u, \partial_{t}^{2} \vartheta, \partial_{t}^{2} q\right]$. Only the summands containing one of the factors $\left(A-\alpha E_{n}\right)$ and $\left(B-\beta E_{n}\right)$ cannot be treated as done in (4.1). But since $(A, B)$ 
is assumed to be smooth enough, one can apply the mean value theorem and conclude

$$
\left|A(\nabla u, \vartheta, q)-\alpha E_{n}\right|=|A(\nabla u, \vartheta, q)-A(0,0,0)| \leq C|(\nabla u, \vartheta, q)|
$$

and $\left|B-\beta E_{n}\right| \leq C|(\nabla u, \vartheta, q)|$, respectively.

Thus, we obtain again

$$
\left|\int_{\Omega} \tilde{F} \Delta u_{|t| t} \mathrm{~d} x\right| \leq C\left(\Lambda^{3 / 2}+\Lambda^{2}\right) .
$$

As a last example we want to estimate a summand of $R_{\mathrm{c}}$ in (3.17) by at least $\sqrt{\Lambda}^{3}$. For this purpose we will use explicitly the boundary condition $\left.\vartheta\right|_{\partial \Omega}=0$ and succeed with partial integration:

$$
\begin{aligned}
& \quad\left|\int_{\Omega}\left(c_{|t| t} \vartheta_{\mid t}+c_{\mid t} \vartheta_{|t| t}+g_{|t| t} \operatorname{div} q+g_{\mid t} \operatorname{div} q_{\mid t}+\operatorname{tr}\left(B_{|t| t} \nabla u_{\mid t}+B_{\mid t} \nabla u_{|t| t}\right)\right) \nabla \vartheta_{|t| t \mid t} \mathrm{~d} x\right| \\
& =\left|\int_{\Omega} \nabla\left(c_{|t| t} \vartheta_{\mid t}+c_{\mid t} \vartheta_{|t| t}+g_{|t| t} \operatorname{div} q+g_{\mid t} \operatorname{div} q_{\mid t}+\operatorname{tr}\left(B_{|t| t} \nabla u_{\mid t}+B B_{\mid t} \nabla u_{|t| t}\right)\right) \vartheta_{|t| t \mid t} \mathrm{~d} x\right| \\
& \leq C \\
& \quad+\left\|\partial_{t}^{2} \nabla c\right\|\left\|\partial_{t} \vartheta\right\|_{\infty}+\left\|\partial_{t}^{2} c\right\|_{L^{4}}\left\|\partial_{t} \nabla \vartheta\right\|_{L^{4}}+\left\|\partial_{t} \nabla c\right\|_{L^{4}}\left\|\partial_{t}^{2} \vartheta\right\|_{L^{4}}+\Lambda^{1 / 2}\left\|\partial_{t} c\right\|_{\infty} \\
& \quad+\left\|\partial_{t}^{2} \nabla g\right\|\|\operatorname{div} q\|_{\infty}+\left\|\partial_{t}^{2} g\right\|_{L^{4}}\|\Delta q\|_{L^{4}}+\left\|\partial_{t} \nabla g\right\|_{L^{4}}\left\|\partial_{t} \operatorname{div} q\right\|_{L^{4}}+\Lambda^{1 / 2}\left\|\partial_{t} g\right\|_{\infty} \\
& \quad+\left\|\partial_{t}^{2} \nabla B\right\|\left\|\partial_{t} \nabla u\right\|_{\infty}+\left\|\partial_{t}^{2} B\right\|_{L^{4}}\left\|\partial_{t} \nabla^{2} u\right\|_{L^{4}}+\left\|\partial_{t} \nabla B\right\|_{L^{4}}\left\|\partial_{t}^{2} \nabla u\right\|_{L^{4}} \\
& \left.\quad+\Lambda^{1 / 2}\left\|\partial_{t} B\right\|_{\infty}\right) \Lambda^{1 / 2} \\
& \leq C
\end{aligned}
$$

As mentioned before, all the appearing perturbation terms can be bounded by $\Lambda^{3 / 2}$ or higher powers of $\Lambda$. Therefore, no other techniques than those used above are required.

Finally, all arguments are presented and we can conclude the proof of Theorem 3.2 .

REMARK 4.2. Actually we did not apply the radial symmetry in the sense of Lemma 2.2 which suggests a transformation of the system to spherical coordinates as done, for example, in [7] for the parabolic system of thermoelasticity. In fact, we just used that the appearing vector fields have symmetric gradients in $\Omega$ and are orthogonal to $\partial \Omega$, i. e. for $n=3$,

$$
\begin{array}{rll}
\operatorname{rot} u=\operatorname{rot} q=0 & \text { in } & {[0, \infty) \times \Omega,} \\
\nu \times q=0 & \text { in } & {[0, \infty) \times \partial \Omega .}
\end{array}
$$

These are exactly the conditions which are made in [12]. In this mentioned paper it suffices to assume that only the initial data satisfy (4.2) and (4.3), for in the linear case then both hold automatically for all times. For the nonlinear equations this conclusion is not possible. Therefore we rather made the assumption 2.3 on the behavior of transformation. Nevertheless, we presented the proof as generally as possible.

Acknowledgement. This work was supported by the Deutsche Forschungsgemeinschaft, DFG-project "Hyperbolic Thermoelasticity" (RA 504/3-1). 


\section{REFERENCES}

[1] Adams, R. A.: Sobolev spaces. Academic Press, New York (1978). MR0450957 (56:9247)

[2] Carlson, D. E.: Linear thermoelasticity. Handbuch der Physik VIa/2, Springer-Verlag, New York (1972).

[3] Cattaneo, C.: Sulla conduzione del calore. Atti Sem. Mat. Fis. Univ. Modena 3, 83-101 (1948). MR.0032898(11:362d)

[4] Chandrasekharaiah, D. S.: Hyperbolic thermoelasticity: a review of recent literature. Appl. Mech. Rev.

[5] Irmscher, T.: Rate of stability in hyperbolic thermoelasticity. Konstanzer Schriften in Mathematik und Informatik Nr. 214 (2006).

[6] Irmscher, T. and Racke, R.: Sharp decay rates in parabolic and hyperbolic thermoelasticity. IMA J. Appl. Math. 71, 459-478 (2006). MR2228916 (2007b:74022)

[7] Jiang, S.: Exponential decay and global existence of spherically symmetric solutions in thermoelasticity. Chin. Ann. Math. 19A, 629-640 (1998, in Chinese). MR1667574 (99m:73005)

[8] Jiang, S. and Racke, R.: Evolution equations in thermoelasticity. Monographs Surveys Pure Appl. Math. 112, Chapman \& Hall/CRC, Boca Raton (2000). MR1774100 (2001g:74013)

[9] Jiang, S., Muñoz Rivera, J.E. and Racke, R.: Asymptotic stability and global existence in thermoelasticity with symmetry. Quart. Appl. Math. 56, 259-275 (1998). MR1622566 (99d:35163)

[10] Leis, R.: Initial boundary value problems in mathematical physics. B. G. Teubner-Verlag, Stuttgart; John Wiley \& Sons, Chichester (1986). MR841971 (87h:35003)

[11] Racke, R.: Thermoelasticity with second sound - exponential stability in linear and non-linear 1-d. Math. Meth. Appl. Sci. 25, 409-441 (2002). MR1888164 (2002m:74016)

[12] Racke, R.: Asymptotic behavior of solutions in linear 2- or 3-d thermoelasticity with second sound. Quart. Appl. Math. 61, Nr. 2, 315-328 (2003). MR1976372(2004a:74026)

[13] Rieger, M. O.: Exponential stability and global existence in thermoelasticity with radial symmetry. Quart. Appl. Math. 62, 1-25 (2004). MR.2032570 (2004j:74056)

[14] Tarabek, M. A.: On the existence of smooth solutions in one-dimensional nonlinear thermoelasticity with second sound. Quart. Appl. Math. 50, 727-742 (1992). MR1193663 (93j:73013) 